\title{
Pepsinogen in gastric carcinoma cells
}

\author{
WA REID*, WD.THOMPSON $\dagger$, J KAY $\ddagger$ \\ From the * University Department of Pathology, Royal Infirmary, Glasgow, the †University Department of \\ Pathology, Aberdeen Royal Infirmary, and the $\ddagger$ Biochemistry Department, University College, Cardiff
}

SUMMARY Immunoperoxidase staining of 125 gastric carcinomas showed pepsinogen in tumour cells of seven $(5.6 \%)$ cases. Carcinomas from other sites were negative. The phenomenon is probably too uncommon to be useful in identifying secondary carcinomas as gastric in origin, but it is of some biological interest.

Pepsinogen has been detected previously in gastric carcinomas 12 and it has been suggested that this could be a useful marker in their study and identification. In the few limited reports published so far pepsinogen has been identified either biochemically or by immunofluorescence on frozen sections. However these techniques are not applicable to most surgical biopsy specimens which are generally fixed. The present study was performed, therefore, to establish whether pepsinogen is detectable in such surgical material by the immunoperoxidase (PAP) technique ${ }^{3}$ and to assess its potential value as a marker specific for tumours arising in the stomach. Commercial pig pepsin proved suitable for raising antisera which cross react with human pepsinogen.

\section{Material and methods}

\section{TISSUES}

All tumour blocks studied were from the surgical routine file (Glasgow Royal Infirmary) and had been fixed in $10 \%$ unbuffered formol saline before paraffin embedding. One hundred and twenty-five consecutive gastric carcinomas were obtained and blocks were chosen, wherever possible, to include adjacent normal mucosa as a positive control. A series of adenocarcinomas from colon (21), breast (18), endometrium (16), ovary (20), prostate (24), gallbladder (1), bile duct (2) and pancreas (2) was also studied. In separate experiments, non-tumour gastrectomy specimens were obtained fresh for the study of effects of autolysis on pepsinogen diffusion.

\section{PRODUCTION OF ANTISERA}

Antiserum A was raised in rabbits by intramuscular injection of $1 \mathrm{mg}$ pig pepsin (Sigma) in complete Freund's adjuvant followed by three injections of 0.5 $\mathrm{mg}$ at two-weekly intervals. The rabbits were bled after a further two weeks. Later an antiserum B was raised in a similar manner against a more highly purified preparation of pig pepsinogen. This was prepared using DEAE-cellulose (Whatman) and Sephadex G100 (Pharmacia) column chromatography as described by Ryle. ${ }^{4}$ The peak of pepsinogen (A) was further purified by affinity chromatography with poly-L-lysine-Sepharose giving a homogeneous pepsinogen as assessed by polyacrylamide gel electrophoresis. ${ }^{5-7}$

\section{SPECIFICITY AND USE OF ANTISERA}

Double diffusion experiments were performed on Ouchterlony agar plates to examine precipitin lines.

The antibody was used on $6 \mu \mathrm{m}$ paraffin sections in the immunoperoxidase enzyme bridge method ${ }^{3}$ at a dilution of $1 / 200$. Sections were counterstained with haematoxylin. Because of the limited amount of pure antigen available for absorption purposes the tumours were first examined with antiserum $A$, the commercial pepsin being used as absorption control in every case. Positive cases were confirmed using antiserum B, absorptions being performed both with purified pepsinogen and pure pepsin. Pure pepsin was prepared by exposing pure pepsinogen to $\mathrm{pH} 2$ for 20 min at room temperature before neutralisation with $\mathrm{NaOH}$. A positively stained section of normal gastric mucosa was impregnated with resin and a selected area peeled off for electron microscopy. ${ }^{8}$

\section{Results}

SPECIFICITY OF ANTISERA FOR PEPSINOGEN

Double diffusion in agar with antiserum $A$ showed a double precipitin line against commercial pepsin, and a single line with purified pepsinogen and pepsin. One of the two lines obtained with antiserum A against the commercial pepsin, showed identity with that obtained against pure pepsinogen. Antiserum B 


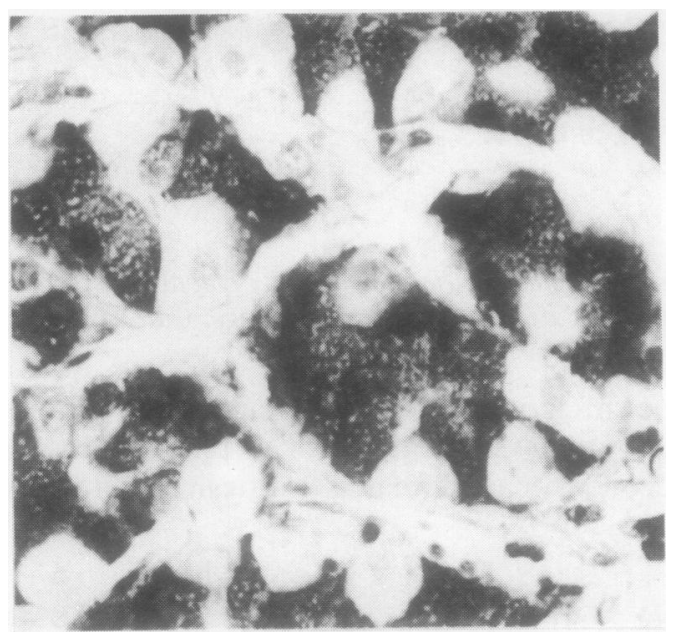

Fig. 1 Pepsinogen staining of chief cells in crypts of normal gastric body. Parietal cells are negative (PAP method).

showed a single line with all of these preparations. There was partial identity between pepsinogen and pepsin.

SELECTIVITY OF STAINING IN NORMAL STOMACH Sections of normal human gastric fundus showed intense granular staining in the chief cells (Fig. 1). In addition weaker staining was observed in a number of cases in the cytoplasm of some of the parietal cells. This was generally homogeneous and fainter than that in the chief cells, but in some cells the staining appeared to be in tubular structures. However, it was observed that this staining was minimal in immediately fixed fresh tissue but became progressively more prominent within minutes if

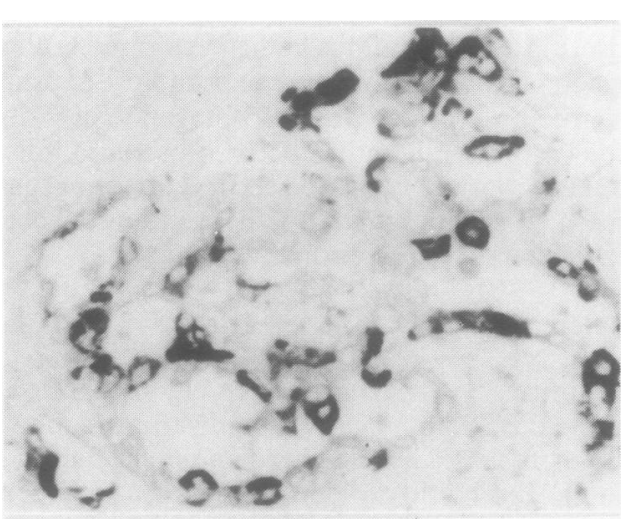

Fig. 2 Pepsinogen in cytoplasm of cells of moderately differentiated adenocarcinoma, stomach. Staining is focal. (PAP method).

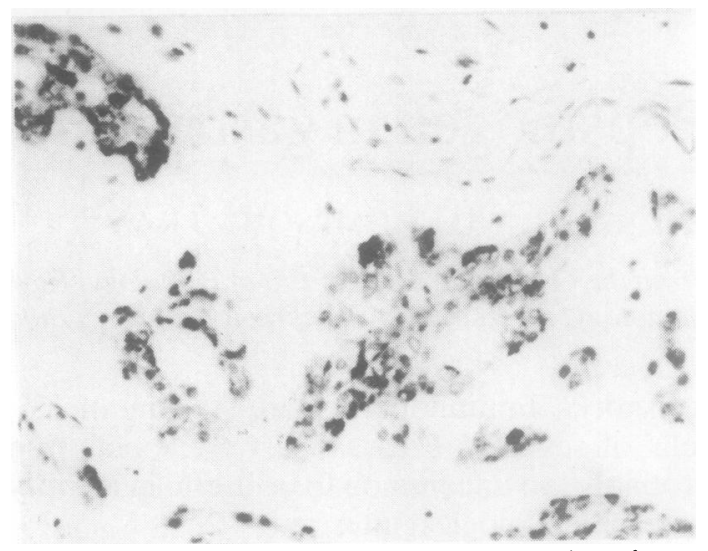

Fig. 3 Pepsinogen in cytoplasm of cells of moderately differentiated adenocarcinoma, stomach. Staining is focal (PAP method).

fixation was delayed. It was probably a diffusion artefact and this was confirmed by electron microscopy of a positively stained area of gastric mucosa from a paraffin section without uranyl acetate treatment; granular reaction product appeared to be associated only with rough endoplasmic reticulum and zymogen granules of chief cells.

Positive staining at light level was abolished by prior absorption of antiserum A with commercial pig pepsin, and of antiserum B by highly purified pepsinogen. The absorptions were performed in 0.05 $M$ Tris-HC1 buffer, $\mathrm{pH} 7.6$ for one hour at room temperature, since pepsin is inactivated rapidly above pH 6. ${ }^{9}$ Inactivated pure pepsin was more efficient at blocking staining by antipepsin (antiserum A) than was pure pepsinogen, and antipepsinogen (antiserum B) was more readily blocked by pepsinogen than pepsin.

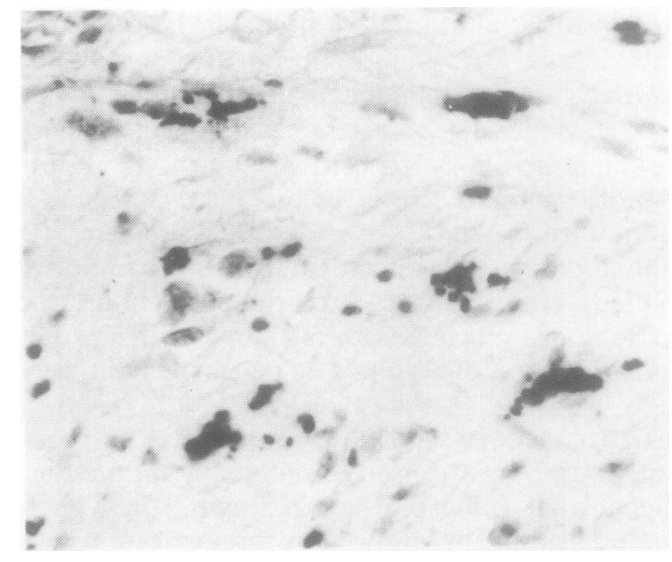

Fig. 4 Pepsinogen in cytoplasm of cells of poorly differentiated carcinoma, stomach (PAP method). 


\section{STAINING OF TUMOURS}

Seven of 125 gastric carcinomas gave unequivocal positive staining with both antisera. In all cases staining was focal. In five moderately differentiated carcinomas it was focal within each tumour and focal in cells within acini (Figs. 2 and 3). In two poorly differentiated carcinomas staining was again focal though present in large numbers of cells in such areas (Fig. 4). All seven carcinomas were positive with antisera A and B and showed the same pattern of staining. As with normal stomach, staining was abolished by prior absorption of antisera with pepsin or purified pepsinogen. All of the adenocarcinomas from other sites were negative with antipepsin (antiserum A) and were not further studied with antiserum B, there being only a limited amount of pure pepsinogen available for blocking purposes.

\section{Discussion}

Although the antiserum A raised against commercial pig pepsin appeared on double diffusion to contain antipepsin and a second antibody of unknown specificity, the pattern of staining in normal stomach and tumours was identical to that obtained with the pure antiserum B to pepsinogen. The unknown antigen may be one of the minor pepsins known to be present in small amounts in commercial pepsin preparations. ${ }^{10}$ The antiserum $B$ raised against purified pig pepsinogen proved to be specific for human pepsinogen as judged by immunodiffusion and selectivity of chief cell staining by the immunoperoxidase method.

This study has shown that pepsinogen is demonstrable by the immunoperoxidase method in both normal gastric mucosa and a small proportion $(5.6 \%)$ of gastric carcinomas. None of a series of non-gastric adenocarcinomas was positive. Therefore, although this method is highly specific for gastric carcinoma and of biological interest, it is not of much diagnostic use in detecting the primary origin of metastatic adenocarcinoma.

This problem does not appear to be one of low sensitivity, since staining is intense where present. Perhaps the small number of tumours stained is simply a reflection of the fact that most gastric carcinomas arise in the pyloric antrum rather than the fundus. The antisera for the present study were raised against pig pepsinogen A which appears to correspond to the main type of pepsinogen found in the human gastric fundus. ${ }^{11}$ Other types of pepsinogen are known to be produced mainly in the antrum and may be immunologically distinct. ${ }^{12} \mathrm{We}$ are attempting to raise antisera to these in the hope of finding a more general marker for gastric carcinoma.

The Lauren classification divides gastric carcinomas into intestinal and diffuse types on histological grounds, ${ }^{13}$ and Lauren has suggested that the aetiology and pathogenesis might be different. Of the seven positively stained tumours in the present study, two were of diffuse, three of intestinal type. This observation supports Morson's criticism of the Lauren classification made on histological and prognostic grounds, ${ }^{14}$ and questions the biological validity of the distinction between intestinal and diffuse types.

We wish to thank Dr J Jass, Department of Histopathology, Central Middlesex Hospital, Acton Lane, London NW10 7NS for classifying the positive tumours by the Lauren system and Dr J Shepherd, Biochemistry Department, Glasgow Royal Infirmary for advice on chromatography.

\section{References}

' Hirsch-Marie H, Loisillier F, Touboul JP, Burtin P. Immunochemical study and cellular localisation of human pepsinogens during ontogenesis and in gastric cancer. Cancer Lab Invest 1976;34:623-32.

${ }^{2}$ Kalinovsky VP, Melnikov PA, Seits IF. Biochemical test for the presence of pepsinogen-pepsin for the diagnosis of stomach cancer metastases. Vopr Onkol 1977;23:18-22.

${ }^{3}$ Mason TE, Taylor CR. The distribution of muramidase (lysozyme) in human tissues. J Clin Pathol 1975;28:124-32.

4 Ryle AP. The porcine pepsins and pepsinogens. Methods Enzymol 1970;19:316-36.

${ }^{5}$ Nevaldine B, Kassell B. Bovine pepsinogen and pepsin IV. A new method of purification of the enzyme. Biochim Biophys Acta 1971;250:207-9.

- Kay J. The $\mathrm{N}$-terminal amino acid sequence of bovine pepsinogen. FEBS Proc Meet Abstract 458, 1972.

' Harboe M, Anderson PM, Foltmann B, Kay J, Kassell B. The activation of bovine pepsinogen.J Biol Chem 1974;249:4487-94.

${ }^{8}$ Hashida Y. Electron microscopic demonstration of viral particles in sudden infant death syndrome. Ultrastruct Pathol 1980;1:710.

${ }^{9}$ Kay J, Valler MJ, Keilova H, Kostka V. Pepsin-like proteinases and their precursors. In: Turk V, Vitale L, eds. Proteinases and their inhibitors-structure, function and applied aspects. Oxford: Pergamon, 1981.

10. Ryle AP. Parapepsinogen II: the zymogen of parapepsin II. Biochem J 1960;75:145.

"Foltmann B, Axelsen NH. Gastric proteinases and their zymogens: phylogenetic and developmental aspects. Enzyme Regulation and Mechanism of Action 1979;60:271-80.

12 Samloff IM, Liebman WM. Purification and immunochemical characterisation of group II pepsinogens in human seminal fluid. Clin Exp Immunol 1972;11:405-16.

${ }^{13}$ Lauren P. The two histological main types of gastric carcinomas: diffuse and so-called intestinal type carcinoma. An attempt at a histoclinical classification. Acta Pathol Microbiol Scand 1965;64:31-49.

${ }^{14}$ Day DW, Morson BC. Gastric cancer. Recent Adv Histopathol No 10 1978:159-72.

Requests for reprints to: Dr WA Reid, Department of Pathology, Royal Infirmary, Glasgow G4 0SF, Scotland. 